\title{
Encadrement des produits et des procédés : réglementation et normalisation du commerce international
}

Oléagineux, Corps Gras, Lipides. Volume 10, Numéro 4, 273-9, JUILLET-AOÛT 2003, Problématiques actuelles dans le domaine de l'analyse des oléagineux et des corps gras

Auteur(s) : Odile MORIN, Coordination scientifique et technique Institut des corps gras -- ITERG rue Monge, Parc Industriel F33600 - Pessac .

Author(s) : Odile MORIN

Résumé : Produits et procédés sont encadrés à la fois par des réglementations et, à un autre niveau, par des normes du commerce international. Cette présentation traite des textes réglementaires au niveau communautaire et national. On rappellera que l'entrée en vigueur d'un règlement européen est suivie d'une transposition dans le droit de chaque pays membre et que la réglementation nationale s'applique en l'absence de dispositions communautaires. En matière de commerce international, seront évoquées les actions de normalisation du Conseil Oléicole International (COI) pour les huiles d'olive et de grignons d'olive et celles du Codex Alimentarius pour les huiles et graisses comestibles. L'ensemble des dispositions réglementaires constitue un cadre englobant les productions de l'amont vers l'aval, à la fois sur un plan vertical (oléagineux, huiles et corps gras, huiles d'olive, margarines, procédés de raffinage) et transversalement (composés organiques volatils, OGM, solvants d'extraction, additifs, contaminants...). Le cas de l'huile d'olive est particulier en ce qu'il bénéficie d'un encadrement au niveau international (normes commerciales COI et Codex Alimentarius), européen et national (réglementation). Le Codex Alimentarius, quant à lui, établit des normes à caractère vertical (huiles végétales, graisses animales, huiles d'olive, matières grasses tartinables...) et horizontal (additifs, résidus de pesticides...). L'essentiel de cet encadrement est résumé dans les tableaux qui illustrent cette contribution.

Summary : Products and certain technological processes are concerned by regulations (European Union and national legislation) and also by international standards (International Olive Council for olive oils, Codex Alimentarius for edible fats and oils). Productions are thus framed by different vertical dispositions (oilseeds, fats and oils, olive oil, margarines...) and more and more by horizontal regulations (environment and volatile organic compounds, GMO, solvents for extraction, additives, contaminants...). This reality results in more complex controls, costs increase and pleads for an optimised communication between suppliers and clients. The development of European regulations related to contaminant concerns creates a real challenge and a permanent debate among analysis experts, and quickens the question of a reasonable balance between caution and reality principles. The specific case of olive oil, significantly regulated and concerned by the standards of the international trade organisations, mainly to maintain a high quality, leads to certain difficulties in application or harmonisation between EU, IOOC and Codex. The composition criteria of the Codex standards for oils and fats trend towards larger variations, reflecting the variety of world-wide productions, but also leading to a weaker relevance of the data. Likewise, the recommended limits 
for the quality criteria may be considered as minimum requirements. These facts plead for the necessity of detailed and precise contractual specifications, complying with the regulatory requirements, to guarantee quality and sanitary security within the supplier $\backslash$ buyer transaction.

Mots-clés : Réglementation, UE, France, normes internationales, Codex Alimentarius, COI

Keywords : Regulation, EU, France, international standards, Codex Alimentarius, IOOC

\section{ARTICLE}

Auteur(s) : Odile MORIN

Coordination scientifique et technique Institut des corps gras - ITERG rue Monge, Parc Industriel F33600 - Pessac

\section{Produits et procédés : encadrement vertical des huiles et des corps gras}

Encadrement de l'amont à l'aval des productions : en dehors de la politique agricole commune qui ne sera pas abordée ici, la Communauté européenne s'est dotée d'un ensemble de textes à caractère vertical, visant l'organisation commune des marchés, le transport maritime des huiles et graisses, ou établissant des spécifications particulières à certains produits (tableau 1).

\section{Tableau 1.}

\begin{tabular}{l|l}
$\begin{array}{l}\text { Directive 76/621/CEE } \\
\text { (20/07/76) }\end{array}$ & $\begin{array}{l}\text { Taux maximal d'acide érucique dans les huiles et graisses en l'état } \\
\text { ou dans les denrées alimentaires en contenant } \rightarrow \text { dès } 1979: 5 \%\end{array}$ \\
\hline et Directive 80/891/CE & $\begin{array}{l}\text { En 1980, méthode communautaire de détermination de la teneur } \\
\text { en C22:1 (acide érucique) }\end{array}$ \\
\hline Directive 96/3 & $\begin{array}{l}\text { Encadrement du transport maritime et liste (positive) de produits } \\
\text { autorisés au chargement précédent une cargaison d'huiles ou de } \\
\text { graisses liquides en vrac }\end{array}$ \\
\hline Décision CE $n^{\circ} 87 / 401,93 / 622$ & $\begin{array}{l}\text { Encadrement spécifique à l'huile d'olive : accords internationaux } \\
\text { approuvés/CEE } \\
\text { classement, dénominations et définitions, commercialisation, } \\
\text { caractéristiques physico-chimiques et organoleptiques }\end{array}$ \\
\hline $\begin{array}{l}\text { Règlement } n^{\circ} 136 / 66 \text { CEE, } \\
\text { modifié }\end{array}$ & \\
\hline $\begin{array}{l}\text { Règlement } n^{\circ} \text { 2568/91 CEE, } \\
\text { modifié }\end{array}$ &
\end{tabular}

Au plan national, les procédés de transformation des huiles et des corps gras et les produits euxmêmes sont encadrés par un ensemble de textes à caractère également vertical, comprenant des dispositions communes et particulières, dont certaines pourraient faire l'objet d'évolutions prochaines (tableau 2). 


\section{Tableau 2.}

- Emploi de qualificatifs et de mentions particulières : utilisation culinaire " pour assaisonnement » ou "pour friture et assaisonnement" selon la teneur en $\alpha-C 18: 3$ (seuil fixé à $2 \%$ ) - Additifs alimentaires autorisés

\section{Perspectives d'évolution :}

- Souhait des pouvoirs publics de mise à jour du décret de 1908 modifié - Souhait des fédérations professionnelles de faire évoluer la législation nationale P Dépôt récent de dossiers visant à :

- supprimer l'obligation des mentions particulières d'utilisation (2\% $\alpha$-C18:3) - lever l'interdiction totale d'emploi de l'huile de lin en alimentation humaine

Les margarines et pâtes à tartiner sont réglementées au niveau national et communautaire (tableau 3).

Tableau 3.

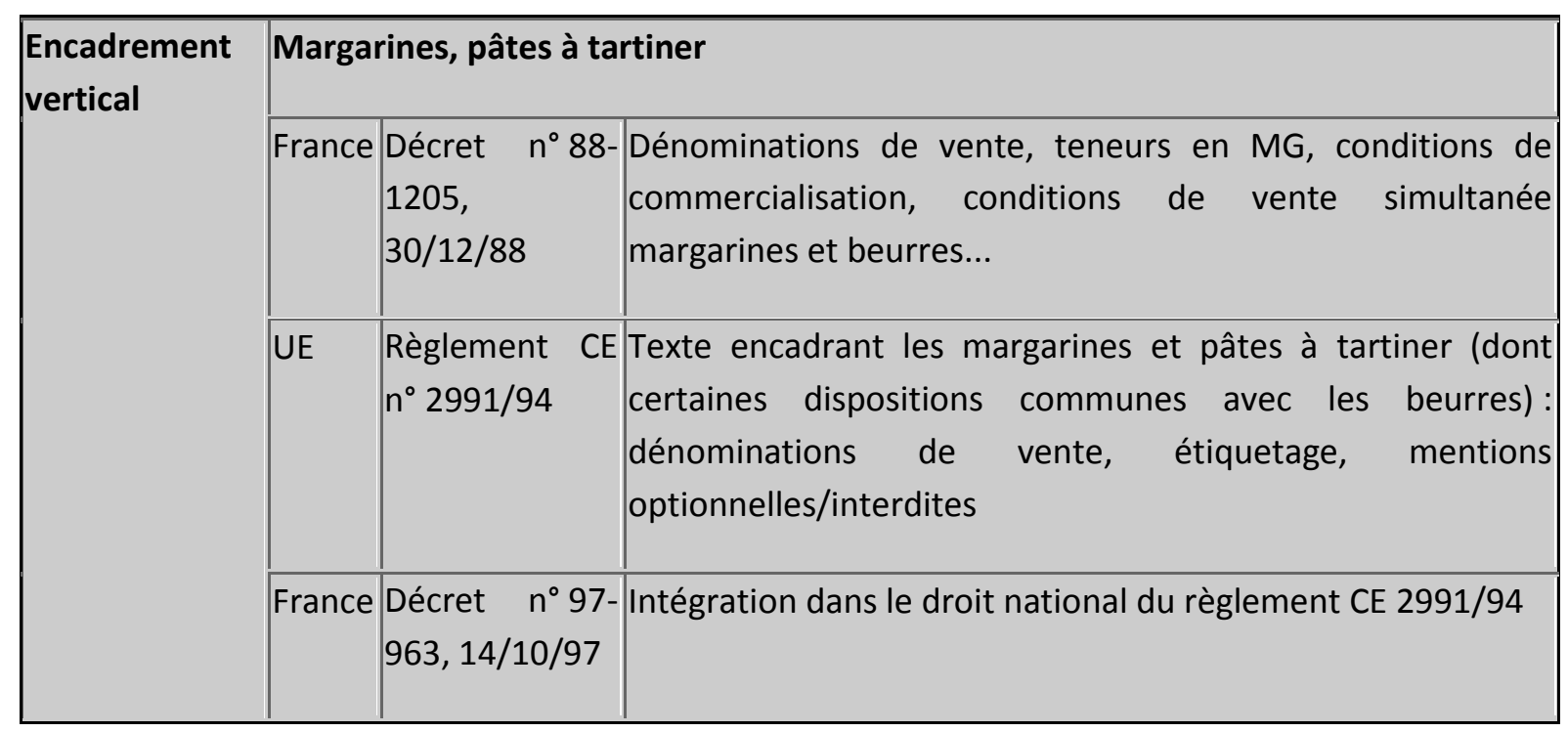

Les corps gras d'origine animale sont concernés, depuis la crise de la vache folle, par une réglementation à caractère vertical encadrant toute une filière de production avec les mesures de protection contre l'agent de l'encéphalopathie spongiforme bovine (ESB), prises au niveau européen et français: interdiction des farines animales, interdiction de certaines graisses animales en alimentation humaine ou animale, obligation de stérilisation des corps gras animaux.

\section{Encadrement par des réglementations à caractère transversal}

Les productions sont également concernées par l'application de textes " horizontaux " : par exemple les dispositions relatives aux composés organiques volatils (COV) dans le cadre de la protection de l'environnement, les solvants d'extraction autorisés (qualité et sécurité sanitaire des produits alimentaires), l'encadrement des organismes génétiquement modifiés (OGM), les autorisations d'emploi des additifs alimentaires, les dispositions fixant des teneurs limites en différents contaminants... (tableaux 4 à 11). 
Tableau 4.

\begin{tabular}{|c|c|c|}
\hline \multicolumn{3}{|c|}{ Réduction des émissions de composés organiques volatils - COV } \\
\hline UE & $\begin{array}{lll}\text { Directive } & 99 / 13 / \text { CE } & \text { du } \\
11 / 3 / 99 & \end{array}$ & $\begin{array}{l}\text { Annexe } 19 \text { : concerne les industries d'extraction d'huiles et corps } \\
\text { gras par la fixation de valeurs limites de consommation d'hexane } \\
\text { par tonne de graine triturée }\end{array}$ \\
\hline France & Arrêté du 29/5/2000 & Transcription de la directive européenne \\
\hline \multicolumn{3}{|c|}{ Solvants d'extraction } \\
\hline UE & $\begin{array}{l}\text { Directive } \quad 88 / 344 / C E E, \\
\text { modifiée par directive } \\
97 / 60 / \text { CEE }\end{array}$ & $\begin{array}{l}\text { Solvants autorisés, critères de pureté et limites maximales } \\
\text { eutorisées dans les denrées ou ingrédients : hexane, } 1 \mathrm{mg} / \mathrm{kg}\end{array}$ \\
\hline France & $\begin{array}{lr}\text { Arrêtés des } & 19 / 11 / 90 \\
12 / 11 / 93 \\
22 / 6 / 98\end{array}$ & Transcriptions de la directive européenne \\
\hline
\end{tabular}

\section{Tableau 5.}

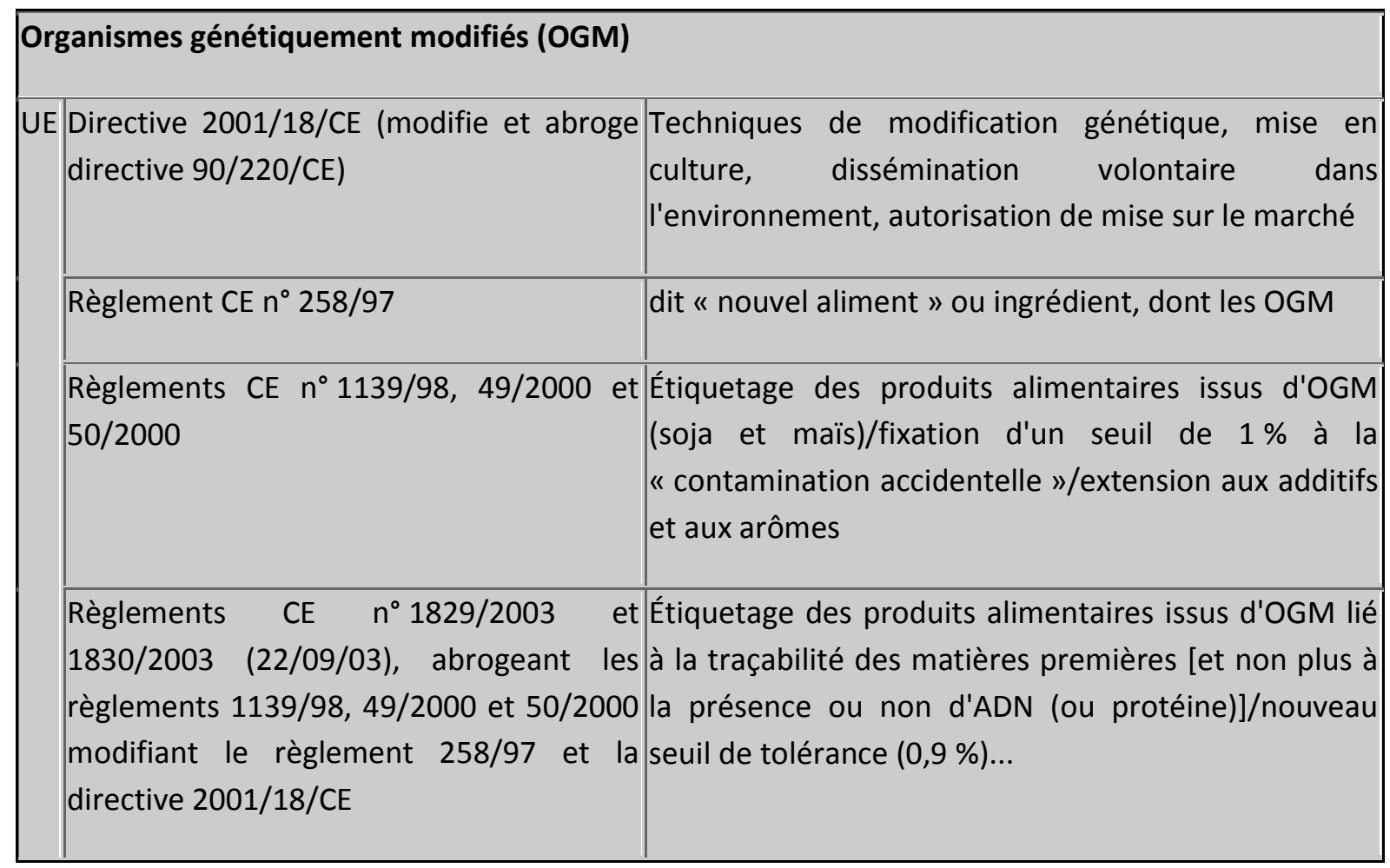


Tableau 6.

\begin{tabular}{|c|c|c|}
\hline \multicolumn{3}{|c|}{ Additifs alimentaires } \\
\hline \multirow[t]{3}{*}{ UE } & \begin{tabular}{|l} 
Directive $89 / 107 /$ CEE modifiée par \\
directive $94 / 34 /$ CE
\end{tabular} & $\begin{array}{l}\text { Directive } \\
\text { Transcrite dans le droit français }\end{array}$ \\
\hline & Directive $94 / 36 / C E$, modifiée & Directive colorants \\
\hline & Directive 95/2/CE, modifiée & $\begin{array}{l}\text { Directive " additifs autres que les colorants et les } \\
\text { édulcorants " }\end{array}$ \\
\hline France & $\begin{array}{l}\text { Arrêtés du 2/10/97, 23/7/98, 22/9/99 } \\
\text { (modifications) }\end{array}$ & $\begin{array}{l}\text { Transposition dans le droit français et } \\
\text { dispositions nationales }\end{array}$ \\
\hline
\end{tabular}

Tableau 7.

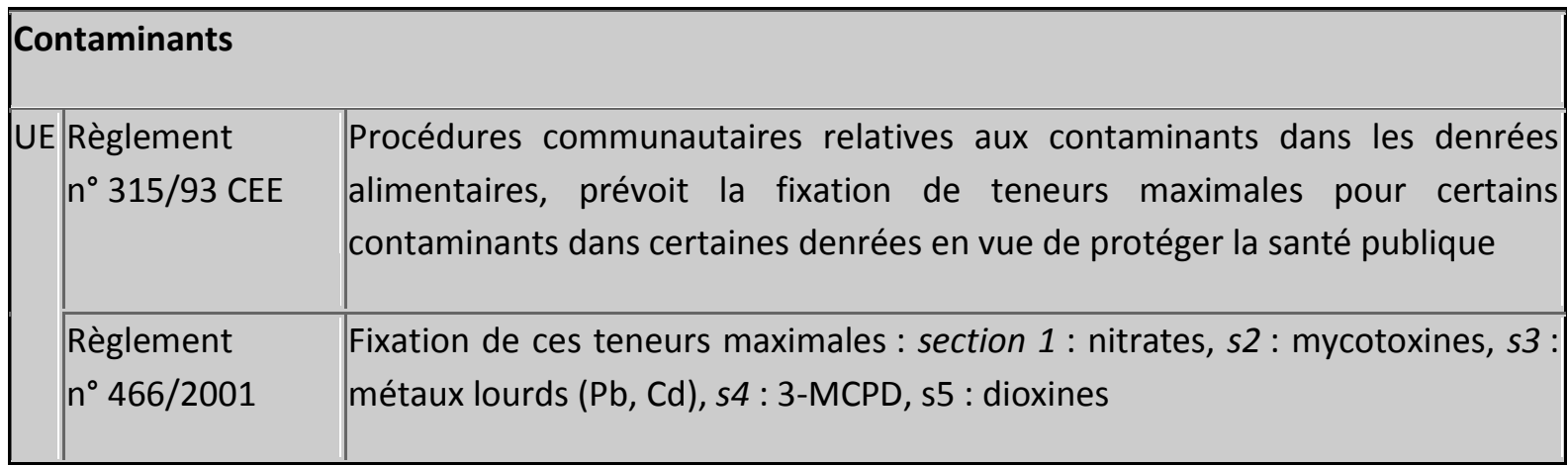

Tableau 8.

\begin{tabular}{|l|l|l|}
\hline Contaminants : pesticides \\
\hline UE & $\begin{array}{l}\text { Directive 91/14/CEE, modifiée } \\
\text { par directive 2001/36/CE }\end{array}$ & Mise sur le marché produits phytosanitaires \\
\hline & $\begin{array}{l}\text { Directive 86/362/CEE modifiée } \\
\text { Directive 90/642/CEE modifiée }\end{array}$ & $\begin{array}{l}\text { Fixation de teneurs maximales en résidus/LMR : oléagineux, } \\
\text { fruits (olives, noix...) }\end{array}$ \\
\hline France & Arrêté du 5/8/92, modifié & $\begin{array}{l}\text { Transcription des directives européennes et dispositions } \\
\text { nationales (dont LMR dans les huiles de colza et de } \\
\text { tournesol) }\end{array}$ \\
\hline
\end{tabular}


Tableau 9.

\begin{tabular}{|c|c|c|}
\hline \multicolumn{3}{|c|}{ Contaminants : mycotoxines } \\
\hline UE & $\begin{array}{l}\text { Règlement } \\
n^{\circ} 466 / 2001 \text { modifié } \\
\text { règlement CE } n^{\circ} 257 / 2002\end{array}$ & $\begin{array}{l}\mathrm{CE} \\
\text { par }\end{array} \begin{array}{l}\text { Arachides, fruits à coques, fruits séchés, produits dérivés de } \\
\text { leur transformation (consommation humaine directe ou } \\
\text { ingrédient) : } \quad \text { aflatoxine } \\
\begin{array}{ll}\mathrm{B} 1+\mathrm{B} 2+\mathrm{G} 1+\mathrm{G} 2<4 \mu \mathrm{g} / \mathrm{kg} & \end{array}\end{array}$ \\
\hline France & Avis du CSHPF, 1990 & $\begin{array}{l}\text { Arachides, pistaches, amandes de bouche ou oléagineux en } \\
\text { général: } \quad \text { aflatoxine } \quad \text { B1 } \\
\text { Huiles vierges ou en mélange avec huiles raffinées : } \\
\text { aflatoxine B1 }<5 \mu \mathrm{g} / \mathrm{kg} \text {, applicable pour les huiles de graines } \\
\text { (ou d'olive) }\end{array}$ \\
\hline
\end{tabular}

Tableau 10.

Contaminants : métaux lourds

UE Règlement CE $\mathrm{n}^{\circ}$ 466/2001du LMR plomb : 0,1 mg/kg dans huiles, matières grasses (MGLA $8 / 3 / 01$ incluses)

LMR cadmium : 0,2 $\mathrm{mg} / \mathrm{kg}$ dans les graines de soja

Tableau 11.

\begin{tabular}{|c|c|c|}
\hline \multicolumn{3}{|c|}{ Contaminants : dioxines } \\
\hline UE & $\begin{array}{l}\text { Règlement CE } n^{\circ} 466 / 2001 \\
\text { modifié par le règlement CE } \\
n^{\circ} 2375 / 2001\end{array}$ & 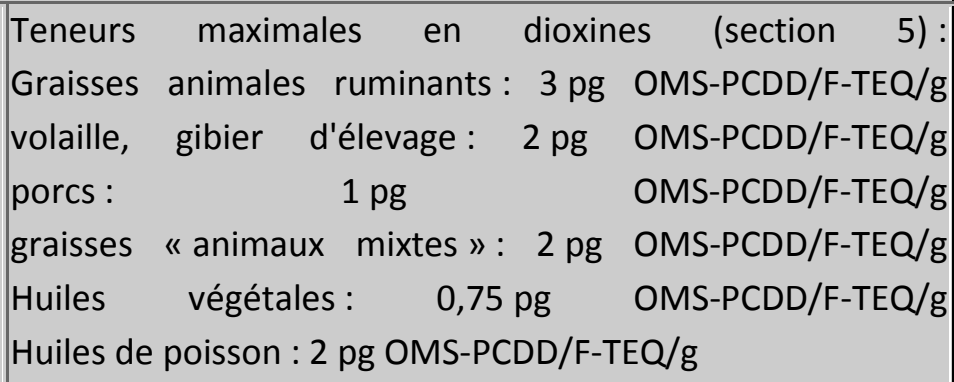 \\
\hline \multicolumn{3}{|c|}{ Contaminants : PCB, suite à la crise de la dioxine de 1999} \\
\hline France & Avis de l'AFSSA, juin 1999 & \multirow{2}{*}{$\begin{array}{l}\text { Dosage de } 7 \text { PCB (congénères) comme approche pertinente } \\
\text { de mise en évidence de contamination dioxine (si > }>, 2 \mathrm{mg} \\
\mathrm{PCB} / \mathrm{kg} \mathrm{MG} \text {, en période de crise) }\end{array}$} \\
\hline UE & $\begin{array}{lll}\text { Décision } & \text { 1999/788/CE } & d u \\
3 / 12 / 99 & & \end{array}$ & \\
\hline
\end{tabular}


Les perspectives réglementaires en matière de contaminants vont nettement dans le sens d'un renforcement, suite aux alertes et aux crises survenues dans un passé proche. Les projets en cours concernent la fixation de teneurs maximales pour les PCB « dioxine-like » et pour les hydrocarbures aromatiques polycycliques (HAP). Ces textes en projet font l'objet de débats parfois contradictoires entre les différents experts de la Commission européenne et ceux des États membres : c'est notamment le cas pour les HAP selon que l'on privilégie une approche multi-composés avec facteurs d'équivalence toxique (par rapport au benzo(a)pyrène, considéré comme le plus toxique) ou le choix de fixer une limite pour le benzo(a)pyrène seul tout en recommandant une surveillance élargie aux autres HAP.

\section{Un encadrement très particulier : l'huile d'olive}

Conseil oléicole international $(\mathrm{COI} / \mathrm{IOOC})$ et normalisation du commerce international : le $\mathrm{COI}$ est une organisation intergouvernementale dont les principales missions sont l'administration des accords internationaux successifs sur l'huile d'olive et les olives de table et de favoriser la coordination internationale des politiques de production, d'industrialisation et de commercialisation du secteur oléicole.

Le tableau 12 résume le contenu de la norme commerciale du COI, applicable à I'huile d'olive et à I'huile de grignons d'olive.

\section{Tableau 12.}

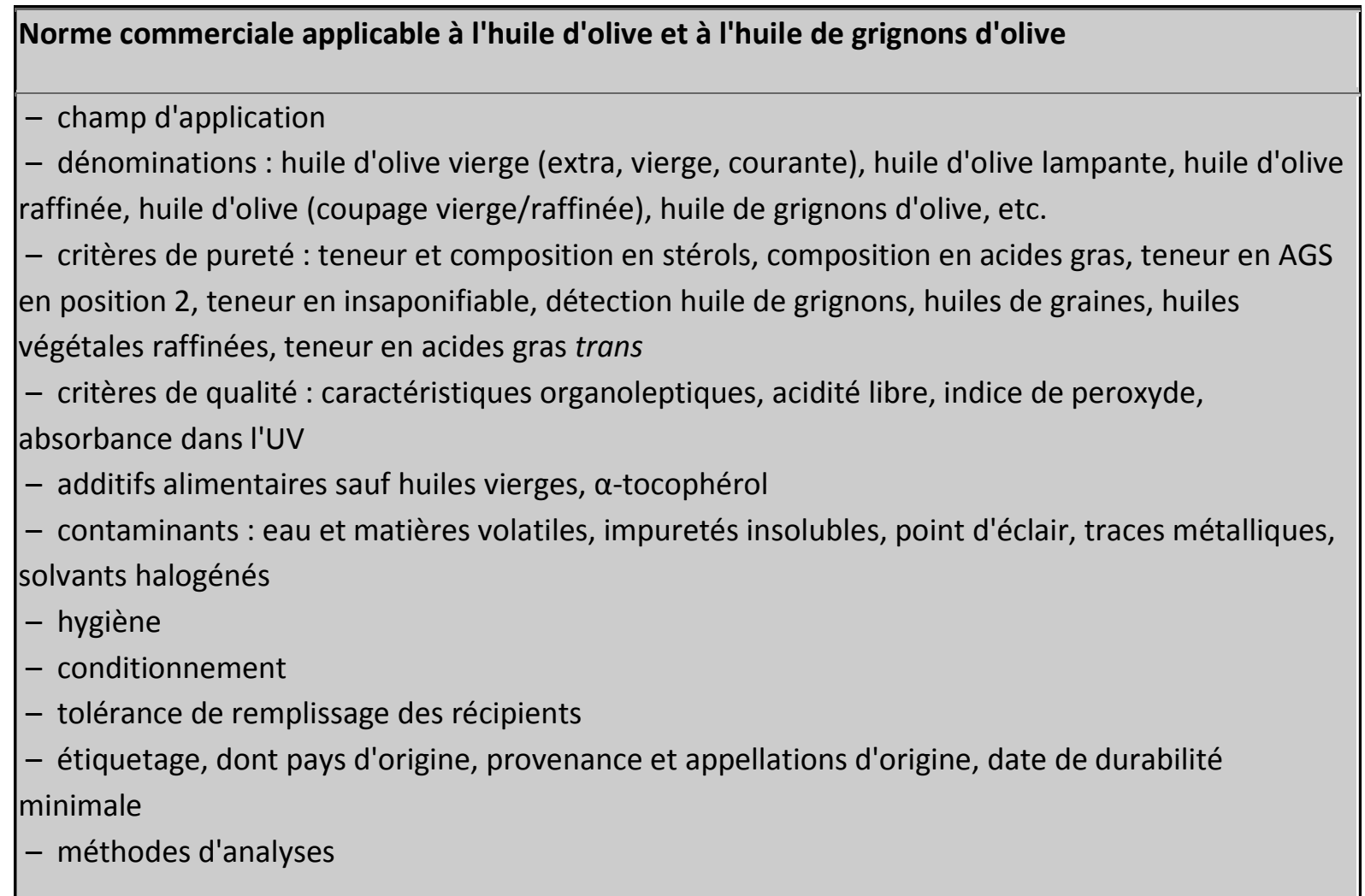

Sur le plan de la normalisation du commerce international, le Codex Alimentarius (voir plus loin) a également édité une norme pour les huiles d'olive. Sa révision en cours tient compte des accords entre le $\mathrm{COI}$ et la Communauté européenne. Au niveau européen, la réglementation 
communautaire encadre significativement les huiles d'olive (tableau 13). Au niveau national, la réglementation intègre la réglementation européenne et fixe par décret les spécifications des olives et des huiles françaises à appellation d'origine contrôlée (AOC) (tableau 14).

Tableau 13.

\begin{tabular}{|c|c|c|}
\hline \multicolumn{3}{|l|}{ Huiles d'olive } \\
\hline \multirow[t]{4}{*}{$\begin{array}{l}\text { Réglementation } \\
\text { européenne }\end{array}$} & $\begin{array}{l}\text { Décision } \\
n^{\circ} 87 / 401, \quad 93 / 622 \\
\text { Règlement } \\
n^{\circ} 136 / 66 \text { CEE, } \\
\text { modifié } \\
\text { Règlement } \\
n^{\circ} 2568 / 91 \text { CEE, } \\
\text { modifié }\end{array}$ & 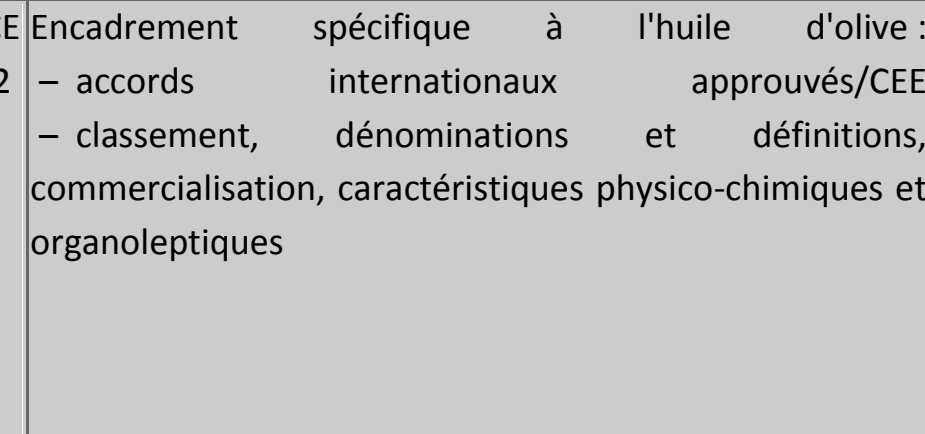 \\
\hline & $\begin{array}{l}\text { Règlement } \\
n^{\circ} 1513 / 2001, \\
\text { 23/7/01 }\end{array}$ & $\begin{array}{l}\text { - Suppression qualité courante } \\
\text { - Acidité vierge extra } \leq 0,8 \mathrm{~g} / 100 \mathrm{~g} \\
\text { - Application }<1 / 11 / 2003\end{array}$ \\
\hline & $\begin{array}{ll}\text { Règlement } & \text { CE } \\
n^{\circ} \text { 2152/2001, } & \\
31 / 10 / 01 & \\
\text { Règlement } & C E \\
n^{\circ} 1019 / 2002, & \\
13 / 6 / 02 & \\
\text { Règlement } & C E \\
n^{\circ} 1964 / 2002 & \\
\text { Règlement } & C E \\
n^{\circ} 1176 / 2003, & \\
1 / 07 / 03 & \end{array}$ & $\begin{array}{l}\mathrm{E} \text { - Indications d'origine : nouvelles dispositions } \\
\text { applicables }<1 / 11 / 02 \text {, sauf produits commercialisés } \\
\text { avant le } 1 / 8 / 02 \\
\text { - Contenances emballages et systèmes d'ouverture, } \\
\text { applicable }<1 / 11 / 03 \\
\text { - Dénominations « pression à froid », " extrait à froid » } \\
\left(27^{\circ} \mathrm{C}\right) \text {, facultatif, }<1 / 11 / 03 \\
\text { - Étiquetage caractéristiques organoleptiques } \\
\text { (facultatif) reporté au } 1 / 11 / 04 \text { et possibilité d'écouler les } \\
\text { stocks (relativement au règlement } 10 / 9 / 2002)\end{array}$ \\
\hline & $\begin{array}{l}\text { Règlement CE } \\
n^{\circ} 796 / 2002,6 / 5 / 02\end{array}$ & E $\begin{array}{lll}\text { - Nouvelle méthode d'analyse sensorielle } \\
\text { - applicable }<1 / 9 / 02\end{array}$ \\
\hline
\end{tabular}


Tableau 14.

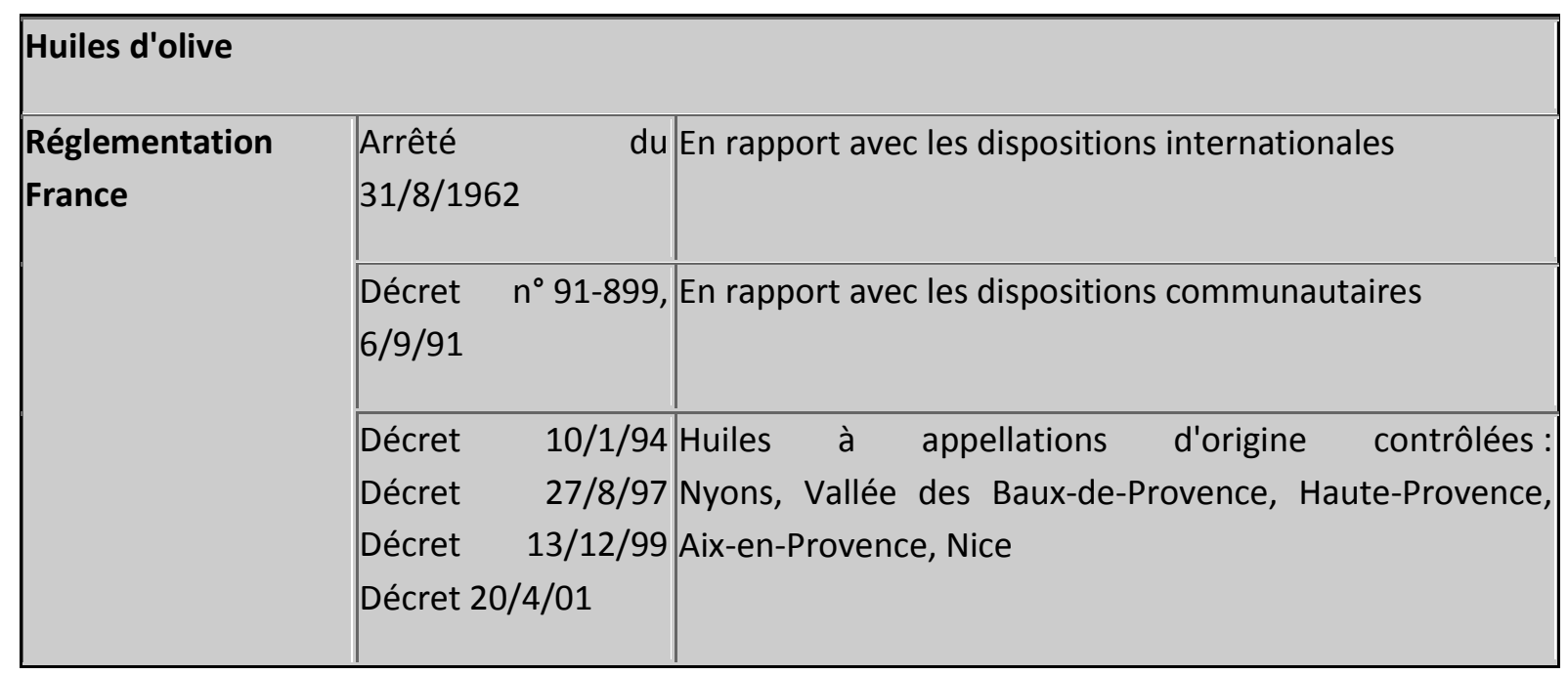

\section{Normalisation du commerce international : le Codex Alimentarius}

Cette organisation, basée à Rome, a été créée en 1962 par la FAO et l'OMS. Son principal objectif est I'harmonisation de normes alimentaires afin de faciliter les échanges internationaux de marchandises et de protéger la santé du consommateur. Les normes homologuées par la Commission du Codex résultent d'un consensus entre experts nationaux et internationaux et ont à ce titre un fondement scientifique et technique. Un Comité vertical huiles et graisses, dont le secrétariat est basé à Londres, travaille régulièrement à la révision ou à la mise en place de nouvelles normes. Pour chaque huile ou graisse visée par une norme, sont définis :

- des critères de composition (acides gras),

- des critères de qualité (dont les indices d'acide et de peroxyde),

- des limites pour certains constituants indésirables ( $\mathrm{Fe}, \mathrm{Cu})$ ou contaminants $(\mathrm{Pb}, \mathrm{As})$.

Les normes en vigueur ou en cours de réexamen sont les suivantes :

- Norme pour les huiles végétales portant un nom spécifique ;

- Norme pour les graisses animales portant un nom spécifique ;

- Norme pour les graisses et huiles comestibles non visées par des normes individuelles ;

- Norme pour les huiles d'olive et de grignons d'olive (en fin de révision depuis février 2003) ;

- Projet de norme pour les matières grasses tartinables, en révision ;

- Code d'usages recommandés pour le transport et l'entreposage des graisses en vrac, en révision. Le tableau 15 résume l'essentiel des caractéristiques physico-chimiques recommandées par les normes Codex relatives aux huiles et aux corps gras. 
Tableau 15.

\begin{tabular}{|c|c|c|c|c|c|c|}
\hline & \multicolumn{6}{|c|}{ graisses Corps gras d'origine animale } \\
\hline & vierges & raffinées & \multicolumn{4}{|c|}{ saindoux suif premier graisse de porc suif } \\
\hline \multicolumn{7}{|c|}{ Facteurs de qualité (annexes des normes) } \\
\hline $\begin{array}{l}\text { Matières volatiles à } \\
105^{\circ} \mathrm{C}(\%)\end{array}$ & \multicolumn{2}{|c|}{ à 0,2} & \multicolumn{4}{|l|}{0,3} \\
\hline $\begin{array}{l}\text { Impuretés insolubles } \\
(\%)\end{array}$ & \multicolumn{2}{|c|}{ es 0,05} & \multicolumn{4}{|l|}{0,05} \\
\hline Teneur en savon (\%) & \multicolumn{2}{|l|}{0,005} & \multicolumn{2}{|l|}{ néant } & \multicolumn{2}{|l|}{0,005} \\
\hline Fer $(\mathrm{mg} / \mathrm{kg})$ & 5,0 & 1,5 & \multicolumn{4}{|l|}{1,5} \\
\hline Cuivre (mg/kg) & 0,4 & 0,1 & \multicolumn{4}{|l|}{0,4} \\
\hline Indice d'acide ou & 4,0 & 0,6 & 1,3 & 2,0 & 2,5 & \\
\hline Acidité (\%) & - & - & 0,65 & 1,0 & 1,25 & \\
\hline Indice de peroxyde & 15 & 10 & 10 & & & \\
\hline \multicolumn{7}{|c|}{ Mémo : limites métaux lourds (corps des normes) } \\
\hline Plomb (mg/kg) & \multicolumn{6}{|l|}{0,1} \\
\hline Arsenic (mg/kg) & \multicolumn{6}{|l|}{0,1} \\
\hline
\end{tabular}

\section{Conclusion}

Ce tour d'horizon, sans avoir la prétention d'être exhaustif, est cependant suffisant pour dégager de grandes tendances et faire les six remarques suivantes pour conclure et orienter la réflexion. - Les normes du Codex International résultant d'un large consensus international évoluent, notamment en matière de critères de composition, vers un élargissement croissant des fourchettes, d'où un manque de pertinence rédhibitoire. De même, les critères de qualité dans ce contexte sont fixés a minima...

- Cette réalité conduit à insister sur l'importance de privilégier les contrats client/fournisseur assortis de cahiers des charges précis, en termes de qualité et de sécurité sanitaire des produits alimentaires. 
- Le cas très particulier de l'encadrement de l'huile d'olive, dont le principal objectif est de garantir la qualité, n'est pas sans poser certaines difficultés d'application (la succession de textes modifiant notamment les dates d'application de certaines dispositions réglementaires traduit cette réalité) et rend plus difficile l'harmonisation entre réglementation et normalisation du commerce international (UE, COI, Codex).

- L'encadrement réglementaire national des procédés et des produits révèle des spécificités qui peuvent poser des difficultés ponctuelles en termes de circulation des produits au sein de l'Union européenne : cette situation est, entre autres, à l'origine des souhaits d'évolution actuels visant à favoriser l'harmonisation entre les différents pays membres de I'Union.

- L'encadrement croissant des productions par des textes à caractère horizontal est une évidence. Cette réalité n'est pas sans conséquences :

- Complexification des systèmes de contrôles

- Augmentation des coûts

- Nécessité d'une communication fondée sur une information "robuste " dans le dialogue client/fournisseur et mise en place indispensable de cahiers des charges adaptés aux produits. - En particulier, le développement accru des textes communautaires relatifs aux contaminants...

- souligne la part prioritaire donnée à la sécurité sanitaire des produits alimentaires,

- constitue un véritable enjeu et une source de débats pour le monde de l'analyse,

- soulève la problématique d'un équilibre raisonnable entre principe de précaution et principe de réalité. $\mathrm{N}$

- interdiction de l'huile de lin

- dénominations : " huile vierge de... ", " huile de...»,

" huile végétale », " préparation à base d'huile... »

Particularité France, Portugal

\begin{tabular}{|c|c|}
\hline \multirow{3}{*}{$\begin{array}{l}\text { Encadrement } \\
\text { vertical }\end{array}$} & Huiles et graisses/procédés de transformation \\
\hline & Dispositions communes \\
\hline & 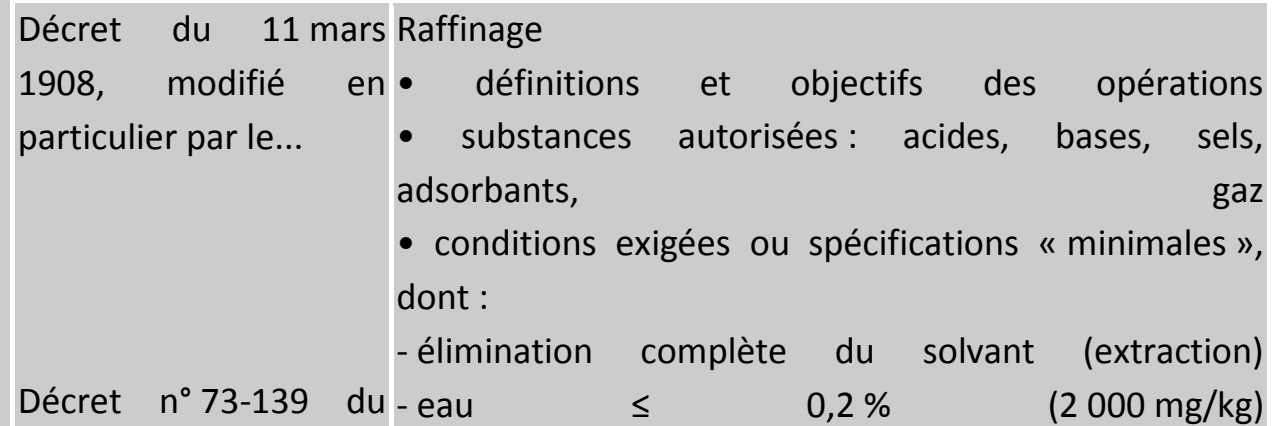 \\
\hline
\end{tabular}




\begin{tabular}{|c|c|c|}
\hline & 12/2/73, modifié & $\begin{array}{l}\text { - savons }<50 \mathrm{mg} / \mathrm{kg} \\
\text { - insoluble dans l'éther de pétrole }<500 \mathrm{mg} / \mathrm{kg}\end{array}$ \\
\hline & & $\begin{array}{l}\text { Modifications: fractionnement, hydrogénation, } \\
\text { interestérification (définitions, auxiliaires autorisés } \\
\text { avec teneurs résiduelles - catalyseurs) }\end{array}$ \\
\hline & & $\begin{array}{l}\text { Caractéristiques } \\
\left.\text { - acide érucique (décret } n^{\circ} 78-840,9 / 8 / 78\right) \\
\text { - composés polaires (décret } n^{\circ} 86-857,18 / 7 / 86 \text { ) }\end{array}$ \\
\hline & & Traitements interdits : estérification, aromatisation \\
\hline & & $\begin{array}{l}\text { Étiquetage : composition graphique pour mélanges } \\
\text { d'huiles } \\
\text { indication de traitement } \\
\text { optionnel : origine }\end{array}$ \\
\hline & & $\begin{array}{l}\text { Contrôles analytiques: } \\
\text { méthodes officielles d'analyse }\end{array}$ \\
\hline Réglementation & Encadrement vertical & Dispositions particulières \\
\hline & & 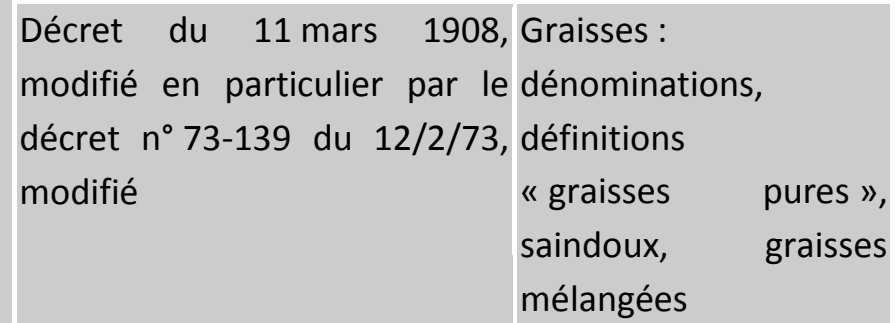 \\
\hline & & Huiles : \\
\hline & & Particularité France, Grèce \\
\hline & ementation européenn & \\
\hline & Politique agricole cor & imune \\
\hline Encadrement & Organisation commu & e des marchés \\
\hline & $\begin{array}{l}\text { Règlement } \\
n^{\circ} 136 / 66 \\
\text { 22/09/1966, modifié }\end{array}$ & $\begin{array}{l}\text { EE Secteurs : graines et fruits oléagineux, matières } \\
\text { du grasses }\end{array}$ \\
\hline
\end{tabular}




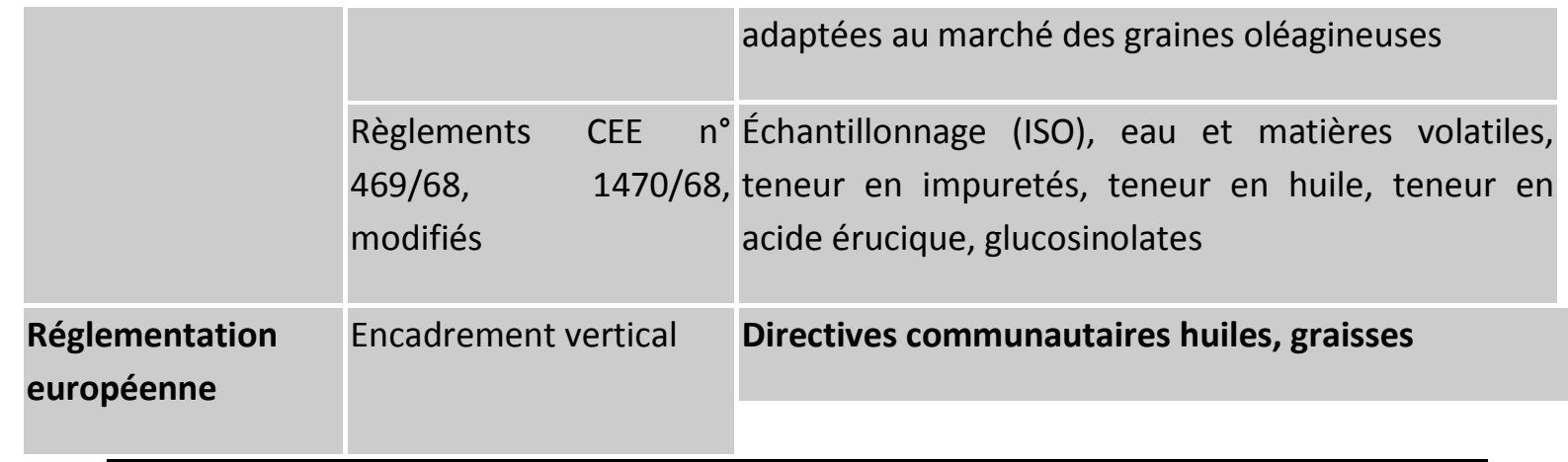

Réglementation européenne 